\title{
REMARKS ON THE OBRECHKOFF INEQUALITY
}

\author{
ALEXANDRE EREMENKO AND ALEXANDER FRYNTOV
}

(Communicated by Franc Forstneric)

Abstract. Let $u$ be the logarithmic potential of a probability measure $\mu$ in the plane that satisfies

$$
u(z)=u(\bar{z}), \quad u(z) \leq u(|z|), \quad z \in \mathbb{C},
$$

and $m(t)=\mu\left\{z \in \mathbb{C}^{*}:|\operatorname{Arg} z| \leq t\right\}$. Then

$$
\frac{1}{a} \int_{0}^{a} m(t) d t \leq \frac{a}{2 \pi},
$$

for every $a \in(0, \pi)$. This improves and generalizes a result of Obrechkoff on zeros of polynomials with positive coefficients.

\section{INTRODUCTION}

Distribution of zeros of polynomials with positive coefficients is an old subject going back to Poincaré [6]. For some recent results we mention [1] and references there.

Obrechkoff [5] proved that for every polynomial $P$ of degree $d$ with non-negative coefficients, and every $\alpha \in(0, \pi / 2)$, the number of roots in the sector $\left\{z \in \mathbb{C}^{*}\right.$ : $|\operatorname{Arg} z| \leq \alpha\}$ is at most $2 \alpha d / \pi$.

A general question about distribution of roots of polynomials with non-negative coefficients was asked by Subhro Ghosh and Ofer Zeitouni [7] in connection with their research on the large deviation theorems for zeros of random polynomials [3].

For each polynomial of degree $d$, we consider the empirical measure which is a probability measure in the plane consisting of atoms of charge $m / d$ at every root of multiplicity $m$. The question of Ghosh and Zeitouni was to describe the closure of empirical measures of polynomials with positive coefficients.

Obrechkoff's inequality implies that every measure $\mu$ in this closure must satisfy

$$
\mu\left\{z \in \mathbb{C}^{*}:|\operatorname{Arg} z| \leq \alpha\right\} \leq \frac{2 \alpha}{\pi},
$$

for every $\alpha \in(0, \pi / 2)$.

A complete description of the closure was given in [2]. It is evident that every polynomial with non-negative coefficients satisfies

$$
|P(z)| \leq P(|z|),
$$

and that the empirical measure of $P$ is symmetric with respect to the real axis.

Received by the editors November 1, 2014 and, in revised form, January 22, 2015 and January $23,2015$.

2010 Mathematics Subject Classification. Primary 30C15, 31A05.

Key words and phrases. Polynomials, logarithmic potential.

This work was supported by NSF grant DMS-1361836. 
For every finite measure $\mu$ in the plane, we define the potential

$$
u_{\mu}(z)=\int_{|\zeta| \leq 1} \log |z-\zeta| d \mu+\int_{|z|>1} \log |1-z / \zeta| d \mu .
$$

Theorem A ([2]). A measure $\mu$ belongs to the closure of empirical measures of polynomials with positive coefficients if and only if $\mu(\mathbb{C}) \leq 1, \mu$ is symmetric with respect to the real axis, and

$$
u_{\mu}(z) \leq u_{\mu}(|z|), \quad z \in \mathbb{C}
$$

Theorem A is proved by approximation of arbitrary potential satisfying (3) and $u(z)=u(\bar{z})$ by potentials of the form $\log |P| / \operatorname{deg} P$, where $P$ is a polynomial with positive coefficients.

Combining Theorem A with Obrechkoff's inequality, one concludes that for every finite measure $\mu$, symmetric with respect to the real line, condition (3) implies (11). The proof of theorem A is complicated, and it is desirable to obtain a direct potential-theoretic proof of the implication (3) $\rightarrow$ (11). Such a proof will be given in this paper. In fact we will prove a stronger statement.

Theorem 1. Let $\mu$ be a probability measure in the plane, symmetric with respect to the real line, whose potential (2) satisfies (3). Then the function

$$
m(t)=\mu\left\{z \in \mathbb{C}^{*}: 0 \leq|\operatorname{Arg} z| \leq t\right\}
$$

satisfies

$$
\frac{1}{a} \int_{0}^{a} m(t) d t \leq \frac{a}{2 \pi}, \quad 0 \leq a \leq \pi .
$$

For the uniform distribution on the unit circle, we have $m(t)=t / \pi$, and equality holds in (5) for all $a$. Obrechkoff's inequality (10) is an immediate corollary of (5): setting $a=2 \alpha$, we obtain

$$
m(\alpha) \leq \frac{1}{\alpha} \int_{\alpha}^{2 \alpha} m(t) d t \leq \frac{2}{a} \int_{0}^{a} m(t) d t \leq \frac{a}{\pi}=\frac{2 \alpha}{\pi} .
$$

Next we discuss the possibility of equality in (1). For the polynomial $P(z)=$ $z^{d}+1$ with non-negative coefficients and $\alpha=\pi / d$, we have equality in (1). Thus (11) is exact for each $\alpha$ of the form $\pi / d, d=2,3,4,5, \ldots$. The second result of this paper is that in fact (1) is best possible for all $\alpha$. For each $\alpha \in(0, \pi / 2)$ we will find a probability measure $\mu$ symmetric with respect to the real axis, satisfying (3) and such that equality holds in (1). Then it follows from Theorem A that the right hand side of (1) cannot be replaced by a smaller number if the resulting inequality must hold for empirical measures of all polynomials with non-negative coefficients.

\section{Proof of Theorem 1}

Without loss of generality we assume that the closed support of $\mu$ is bounded and does not contain 0: it was shown in 2] that arbitrary finite measure satisfying (3) can be approximated by a measure with such a support which also satisfies (3).

Then it is sufficient to consider a potential of the form

$$
u(z):=\int_{\mathbb{C}} \log |1-z / \zeta| d \mu(\zeta),
$$

which differs from (2) by an additive constant, and hence, also satisfies (3). 
For a fixed $\rho \in(0,1)$, consider the function

$$
v_{\rho}(z)=\int_{0}^{\infty} u(z / t) t^{\rho-1} d t
$$

This function is subharmonic and homogeneous,

$$
v_{\rho}(\lambda z)=\lambda^{\rho} v_{\rho}(z), \quad \text { for every } \quad \lambda>0,
$$

therefore it has the form

$$
v_{\rho}\left(r e^{i \theta}\right)=r^{\rho} h_{\rho}(\theta) .
$$

To relate $h$ with $\mu$, we need the integral

$$
\int_{0}^{\infty} \log \left|1-\frac{z}{t}\right| t^{\rho-1} d t=c_{\rho} r^{\rho} \cos \rho(\theta-\pi), \quad z=r e^{i \theta}, 0 \leq \theta \leq 2 \pi,
$$

where $c_{\rho}=\pi /(\rho \sin \pi \rho)$. To see that (8) holds, we notice that the left hand side is a homogeneous subharmonic function of degree $\rho$, so it has the form (7). This function is harmonic in the complement of the positive ray, so it has the form as in the right hand side of (8). To find $c_{\rho}$ we plug $z=-1$ into (8).

Let us define $\phi_{\rho}$ as the $2 \pi$-periodic extension of $\cos \rho(\theta-\pi), 0 \leq \theta \leq 2 \pi$. Then we have

$$
v_{\rho}\left(r e^{i \theta}\right)=\int_{0}^{\infty} \int_{\mathbb{C}} \log \left|1-\frac{r e^{i \theta}}{t \zeta}\right| d \mu(\zeta) t^{\rho-1} d t=c_{\rho} r^{\rho} \int_{\mathbb{C}} \phi_{\rho}(\theta-\arg \zeta) \frac{d \mu(\zeta)}{|\zeta|^{\rho}} .
$$

Comparing this with (7), we obtain

$$
h_{\rho}(\theta)=\int_{\mathbb{T}} \phi_{\rho}(\theta-t) d \nu_{\rho}(t),
$$

where $\mathbb{T}=\mathbb{R} /(2 \pi \mathbb{Z})$ is the unit circle, and

$$
\nu_{\rho}(E)=c_{\rho} \int_{\arg \zeta \in E} \frac{d \mu(\zeta)}{|\zeta|^{\rho}},
$$

for every Borel set $E \subset \mathbb{T}$. When $\rho \rightarrow 0, \nu_{\rho} / c_{\rho} \rightarrow \nu_{0}$, where $\nu_{0}$ is proportional to the radial projection of the measure $\mu$, so $m(t)=\nu_{0}[-t, t]$.

Inequality (3) and symmetry $u(z)=u(\bar{z})$ imply that

$$
2 h_{\rho}(0)-h_{\rho}(a)-h_{\rho}(-a) \geq 0, \quad a \in[0, \pi] .
$$

Using the expression (9), we conclude that

$$
\int_{\mathbb{T}} J_{\rho}(t) d \nu_{\rho}(t) \geq 0
$$

where

$$
J_{\rho}(t)=2 \phi_{\rho}(t)-\phi_{\rho}(t-a)-\phi_{\rho}(t+a) .
$$

Now we divide by $\rho^{2}$ and pass to the limit $\rho \rightarrow 0$, using $\cos t \sim 1-t^{2} / 2$. A simple direct computation shows that $J_{\rho} / \rho^{2} \rightarrow J$, where

$$
J(t)= \begin{cases}2 \pi|t|-2 \pi a+a^{2}, & |t| \leq a, \\ a^{2}, & a<|t| \leq \pi .\end{cases}
$$

Notice that $J_{\rho}$ is $2 \pi$-periodic and satisfies the distributional equation

$$
J_{\rho}^{\prime \prime}+\rho^{2} J=2 \rho \sin \pi \rho\left(2 \delta_{0}-\delta_{a}-\delta_{-a}\right),
$$


where $\delta$ 's are $2 \pi$-periodic delta functions. Therefore $J$ is a $2 \pi$-periodic solution with zero average of the distributional equation

$$
J^{\prime \prime}=2 \pi\left(2 \delta_{0}-\delta_{-a}-\delta_{a}\right),
$$

where the $\delta$ 's are the $2 \pi$-periodic delta functions. This property defines $J$ uniquely, which permits us to write it without any computation.

We conclude that

$$
\int_{0}^{\pi} J(t) d \nu_{0}(t) \geq 0, \text { and thus } \int_{0}^{\pi} J(t) d m(t) \geq 0 .
$$

Integrating the last integral by parts, we obtain

$$
2 \pi \int_{0}^{a} m(t) d t \leq J(\pi) m(\pi)=a^{2},
$$

which is equivalent to (5).

\section{EXAMPLE}

In this section, for any given $\alpha \in(0, \pi / 2)$, we construct a probability measure $\mu$ symmetric with respect to the real line, and satisfying (3), such that Obrechkoff's inequality (10) holds with equality.

Inequalities (6) suggest that the sectors $|\operatorname{Arg} z|<\alpha$ and $|\operatorname{Arg} z| \in(\alpha, 2 \alpha)$ must be free of the measure.

Potential

$$
u(z):=\log \left|z^{2}+1\right|
$$

satisfies (3), and its total Riesz' measure equals 2 . Take $\alpha \in(0, \pi / 2)$ and define the function

$$
u_{\alpha}(z):= \begin{cases}u\left(z^{\pi /(2 \alpha)}\right), & |\operatorname{Arg}(z)|<2 \alpha, \\ u\left(|z|^{\pi /(2 \alpha)}\right), & \text { otherwise. }\end{cases}
$$

This function is subharmonic (its Laplacian will be computed below). It is clear that $u_{\alpha}$ satisfies (3). Let $\lambda_{\alpha}$ be the Riesz' measure of $u_{\alpha}$. One should notice that $\lambda_{\alpha}$ is supported on the set

$$
\{z:|\operatorname{Arg}(z)| \geq 2 \alpha\} \cup\left\{e^{i \alpha}\right\} \cup\left\{e^{i \alpha}\right\} .
$$

Notice that $\lambda_{\alpha}\left\{e^{ \pm i \alpha}\right\}=1$, and $\lambda_{\alpha}$ is absolutely continuous on $\{z:|\operatorname{Arg}(z)| \geq 2 \alpha\}$ with respect to the plane Lebesgue measure, and its density is

$$
\rho_{\alpha}=\frac{1}{2 \pi} \Delta u_{\alpha} .
$$

Since $u_{\alpha}\left(e^{i \theta}\right)$ does not depend on $\theta$ for $|\theta| \in(2 \alpha, \pi)$, we compute the Laplacian $\Delta u_{\alpha}$ in polar coordinates $\left(z=r e^{i \phi}\right)$ as follows:

$$
\begin{aligned}
\rho_{\alpha}\left(r^{i \phi}\right) & =\frac{1}{2 \pi r} \frac{\partial}{\partial r}\left(r \frac{\partial u_{\alpha}}{\partial r}\right)=\frac{1}{2 \pi r} \frac{d}{d r}\left(r \frac{d}{d r} \log \left(1+r^{\pi / \alpha}\right)\right) \\
& =\frac{1}{2 \alpha r} \frac{d}{d r}\left(\frac{r^{\pi / \alpha}}{1+r^{\pi / \alpha}}\right) .
\end{aligned}
$$

Thus,

$$
\left.\lambda_{\alpha}\{z:|\operatorname{Arg}(z)| \geq 2 \alpha\}\right)=\frac{(2 \pi-4 \alpha)}{2 \alpha} \int_{0}^{\infty} r \rho_{\alpha}(r) d r=\frac{\pi-2 \alpha}{\alpha}
$$


and

$$
\lambda_{\alpha}\{\mathbb{C}\}=2+\frac{\pi-2 \alpha}{\alpha}=\frac{\pi}{\alpha} .
$$

Then we define normalized measure $\mu_{\alpha}:=\lambda_{\alpha} / \lambda_{\alpha}(\mathbb{C})$, and

$$
\mu_{\alpha}\left\{e^{ \pm i \alpha}\right\}=\frac{\alpha}{\pi}
$$

So the measure $\mu_{\alpha}$ satisfies the equation

$$
\mu_{\alpha}\{|\operatorname{Arg}(z)| \leq \alpha\}=\frac{2 \alpha}{\pi} .
$$

\section{ACKNOWLEDGEMENT}

The authors thank the referee for valuable remarks.

\section{REFERENCES}

[1] R. W. Barnard, W. Dayawansa, K. Pearce, and D. Weinberg, Polynomials with nonnegative coefficients, Proc. Amer. Math. Soc. 113 (1991), no. 1, 77-85, DOI 10.2307/2048441. MR 1072329 (91k:30007)

[2] Walter Bergweiler and Alexandre Eremenko, Distribution of zeros of polynomials with positive coefficients, Ann. Acad. Sci. Fenn. Math. 40 (2015), no. 1, 375-383, DOI 10.5186/aasfm.2015.4022. MR3310090

[3] S. Ghosh and O. Zeitouni, Large deviations for zeros of random polynomials with i.i.d. exponential coefficients, arXiv:1312.6195.

[4] B. Ja. Levin, Distribution of zeros of entire functions, Revised edition, Translations of Mathematical Monographs, vol. 5, American Mathematical Society, Providence, R.I., 1980. Translated from the Russian by R. P. Boas, J. M. Danskin, F. M. Goodspeed, J. Korevaar, A. L. Shields and H. P. Thielman. MR589888 (81k:30011)

[5] N. Obrechkoff, Sur un problème de Laguerre, C. R. Acad. Sci. (Paris) 177 (1923), 102-104.

[6] H. Poincaré, Sur les équations algébriques, C. R. Acad. Sci. 97 (1884), 1418-1419.

[7] O. Zeitouni, Zeros of polynomials with positive coefficients, http://mathoverflow.net/ questions $/ 134998$.

Department of Mathematics, Purdue University, West Lafayette, Indiana 47907

Physical-Engineering Institute of Low Temperature, National Academy of Sciences of Ukraine, Kharkov 310164, Ukraine 